\title{
$-106-$
}

\section{Bemerlungen úber Kali hydrijodinicum.}

\author{
Bon
}

(5. Firnbaber unb DR. Şeder, ber 3eit in Şalle.

Durd bas Miplingen ber Seteitung beb hydriobin: fauren Sults, wurbsn toir verankst, einige Berjuthe jut Zuffinoung bet verbinbernben Urfache anjuftellen, und theilan die erbaltenen Siejultate hier im Folgenden fúçlid) mit.

Mi: nabmen, um Kali hydrijodinicum nad ber von $\mathfrak{B a u p}$ und 5 a illot angegebonen Metbode zu bes rsiten, eine balbe Hnje Sobin, fohitteten diejplbe mit zivei Dradomen Fifenfille in cin Giefié, und golfen

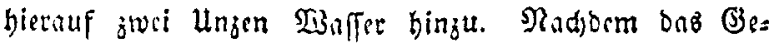
inemge einige 3itit in Berbbrung givelen, brmerften wir blos sine rotbbraune Firbung der Zlafifgerit, obne oas fith jrboch bie minbefte $\mathfrak{B}$ arme babei entivideite, unb feibft nad mebreten Gtumben brmoteten vit Erine Far= beninberung. Dait fegten nun bie Fluffigfeit siner mi= figrn Trmperatur von obngrffigr $50^{\circ} \Re$. aus, unb falbf nath breiftundiger Digeftion fand feine Farbenán= Derung ftatt. Sefet brad)ten wir bie Flüfigfsit jum Sirben, wobsi fith fortwibrent Sobine in violetten Dimpfen verfludtigte, obne oaß (id) bie Fluffigfeit snt= firbte. Eine betridutide Menge Sodine wurbe auf

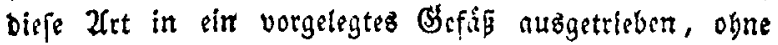

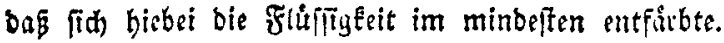

Da baz Entfácben ber F(üfïgEeit burd) Şike nicht gu bewirken war, glaubten wir es ver Unreinigfeit bes Eifens jufdreiben ju mirfen. Wir guben baber not) 
zroei Draddmen Eifen binzu, und auth bann erfolgte weber 13 irmentwidelung not Entfitbung.

Da nun auth be: neue 3 ujas bon (Eifen nid)t bats allf wirkte, glaubten wir mit verfirfoter Jobine geats beitet ju buben. Wit erbiģton onher Sobine in einer

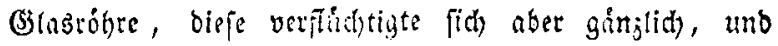

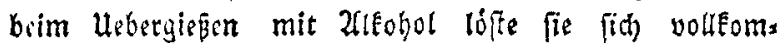
men auf.

Da wir uns nun burd biefe beiden Proben von Der Neinfeit ber Sobine uborjouyt batten, blisb uns

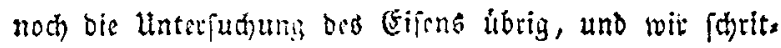
ten baher zu folyenoen Berfutyon.

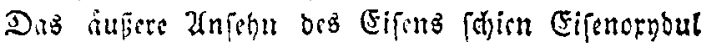
im briyemengten Buftunde ju verrathen.

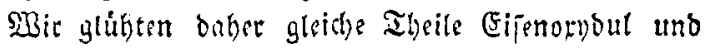

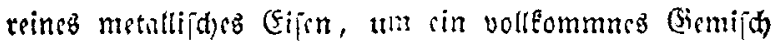

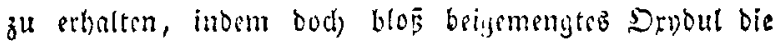
Brabinoung nidt bindern fann. 20 Gran Jodine wult: ben mit rben fo vid bes erbaltenen (Eifens in einem Bhafe mit $23 a$ fer ůbergoffen, es entfrent biebei augen= bliçlid) sine rothbraune Firbung ber Flúliligket, an Ers bişen Derfelben war aber feltft nach einer balben Stunde nidht jut benfen. Wir erbikzten bns Germenge baher bis $z^{4} 5^{\circ} \Re$, und nach breiftunbiger Digefion erfolgte Eeine Entfátbung. Wir exhititen nun ettons yon ber abgegoftenen Flúfirgkeit bis zum Sirben, wobei firth aus= genblictilic bie Sobine verfluthtigte, unb aud bann er= folgte Eine Entfirfuny.

Zus biefem Berfudie foheint hervorjugehen, baß (Eifen mit Eifenornoul vermifdt, fich mit oet Sobine nicht ju Dem horiodinfaren Eifen verbinde. 


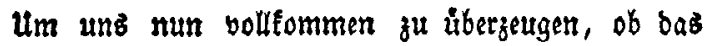
Miślingen unjers Práparatz vom oben angegebnen um= ftanbe abgänge, ober ob eb viefleitht andern Şinder: nifien unterworfen fen, war es nóthig, unjer Eifen felbjt zut prúffer.

Bir übergoffen zu bem Enbe eine beffimmte Duan= titåt unfers Eifens mit Salzfaure, fergten biez siner

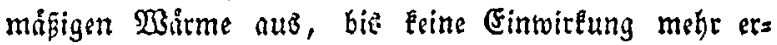
folgte, bann goffert wir bie Flúfligefeit ab, unb fegeten fo oft neue Sáure wieber bingu, biz diefe gar nidyt megr auf den Rúdeftand eintwirte. Rum goffen twit bie

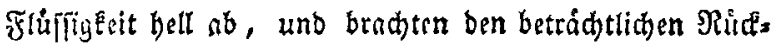
ftano, ben bie Salzläure hintertafien hatte, auf ein Fils ter, laugten biefen gebórig mit Baffer aus, uno trock: neten ferbigen. Diefer Rudffrno verbialt fid bet der unterfudyung wie Rohle mit etwas unteiner Ritefelerbe gemengt.

$\mathfrak{u m}$ nun zu fehn, ob sohle wirtlich im Stande fen bie Berbinbung zlu verbindern, wurbe ein Gemenge aus zwei Theilen Fifen uno einem Thrile Soble eine halbe

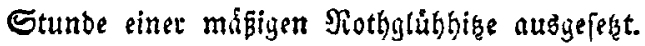

Bon biefem Roblencifen wurben 50 Gran mit eben to viel Sodine in einem Befófer mit ber nơthigen Menge Mlaffer ibbergoffer. Die Flúffigeneit fórbte fidh augens bliálich rothbraun, obne dap fie fidi im mindeften ers wirmte, und aud auf ben surfas von 50 (Stan bes Rohleneifens unb nadherigem Erhigen erfolgte feine

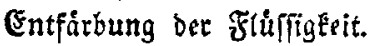

(Fin anbrer $\mathfrak{B e r}$ uch mit 3 Theilen Graphit uno 1 Iheile Sodine, gab bie nåmlidjen Refultate.

Ein Minimum von reinem metallichen Eifen zu 


\section{- $109-$}

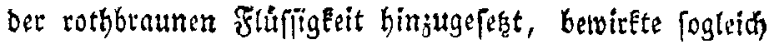

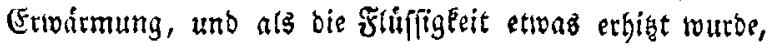

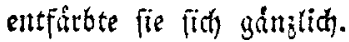

Diefe renigen Berfude folstinen zu betweifen, das (EiTen unb Eifenorybul, wis anth (Fifm und Rohle, bei= bes im gemilchten 3uitande, nid)t im Stande find, eine volleommne Berbinburg mit Der Sobine einjugeben; und bas bei unferm Eifar bie Fohle bie verbindernde ur rache (en. *)

\section{Werfditedene pfarmacentifde \$emerfungen.}

\section{Bont}

Şrovifor Siófeler in Ripplfadt.

\section{I.}

Drfhon ber würoige Weerr Witting uns mit

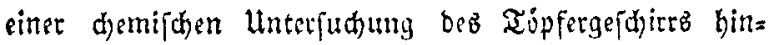

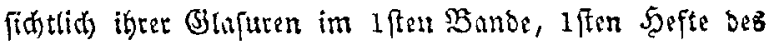

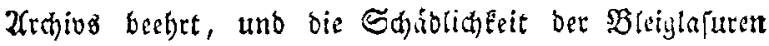
im 2llgemeitren belviefen hat, fo fey es mir bennod et: laubt, befonders, on if ben Gebrauch biefes Gejchirts

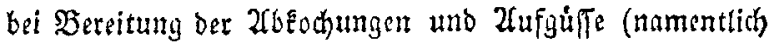
Decoctum Tamarindorum etc.) in einigen 2fpothe= Een leiber angenjheinlidy vernommen, nadfethende $\mathfrak{U}_{\mathfrak{n}}=$ terfud)ung zut Marnung mitzutheilen. -

Einige $U_{\mathfrak{n}}$ en Pulpa Tamarindorum wutben in einem neuen vorther gereinigten hejilịhen \$opfergefhitre bireitet.

Erfter

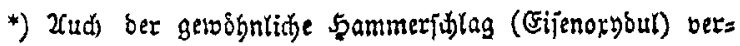
Gielt fid jo.

şandes. 